analyses were performed to determine the association between the infections and clinical manisfestations.

Results The prevalence of $M$. genitalium infection at urethral, rectal and pharyngeal sites was $17.2 \%$ (95\% CI: $13.4 \%$ to $21.0 \%$ ), $11.8 \%$ (95\% CI: $8.4 \%$ to $15.2 \%$ ), and $13.5 \%$ (95\% CI: $9.9 \%$ to $17.1 \%)$, respectively. C trachomatis was more commonly detected in rectum $(16.0 \%, 95 \%$ CI: $12.2 \%$ to $19.8 \%)$ than in urethra $(9.4 \%, 95 \%$ CI: $6.4 \%$ to $12.3 \%)$ and in pharynx $(0.8 \%, 95 \% \mathrm{CI}: 0.1 \%$ to $1.6 \%)$. Urethral $M$. genitalium infection was significantly associated with urethral discomfort in the past 3 months (AOR: 2.22, 95\% CI: 1.09-4.52) and polymorphonuclear leucocyte (PMNL) counts per high-power microscope field (AOR: 2.40, 95\% CI: 1.02-5.62). Rectal $M$. genitalium infection was independently associated with rectal discharge in the past 3 months (AOR: 6.06, 95\% CI: 1.5923.11). For $C$ trachomatis infection, PMNL counts per highpower microscope field (AOR: 4.66, 95\% CI: 1.80-12.07) and having receptive anal intercourse with a male in the past 3 months (AOR: 2.27, 95\% CI: 1.14-4.54) were associated with urethral and rectal $\mathrm{C}$ trachomatis infection, respectively.

Conclusion High prevalence of $M$. genitalium infection was observed among MSM in China at urethral, rectal and pharyngeal sites. M. genitalium infection was significantly associated with urethral and rectal symptoms. C trachomatis was more commonly detected in rectum and more likely to be asymptomatic.

Disclosure of interest statement No potential conflicts of interest.

\section{O03.4 WHAT EXPLAINS ANORECTAL CHLAMYDIA DETECTION IN WOMEN: IMPLICATIONS FOR CONTROL STRATEGIES}

${ }^{1}$ Janneke CM Heijne*, ${ }^{2,3}$ Geneviève AFS van Liere, ${ }^{2,3}$ Christian JPA Hoebe, ${ }^{1}$ Birgit HB van Benthem, ${ }^{2,3}$ Nicole HTM Dukers-Muijrers. ' ${ }^{1}$ entre for Infectious Diseases Control, National Institute for Public Health and the Environment, Bilthoven, The Netherlands; ${ }^{3}$ Department of Medical Microbiology, School of Public Health and Primary Care (CAPHRI), Maastricht University Medical Center (MUMC+), Maastricht, Netherlands; ${ }^{2}$ Department of Sexual Health, Infectious Diseases and Environmental Health, South Limburg Public Health Service, Geleen, Netherlands

\subsection{6/sextrans-2015-052270.94}

Introduction Anorectal Chlamydia trachomatis (Ct) testing in women is not standard. In some countries, women are being tested based on reported anal intercourse. However, universal anorectal Ct testing in STI clinics revealed prevalences over $10 \%$, irrespective of anal intercourse, and showing high cooccurrence with urogenital infections. To inform control strategies in women, this study explores different transmission mechanisms that can explain the high observed Ct prevalences using mathematical modelling.

Methods We developed a pair compartmental model of heterosexuals aged 15-29 years. To inform the model, data on anorectal and urogenital infections are used from heterosexual men and women attending STI clinics. In the model, women can have urogenital and anorectal infections, men only urogenital infections. At all sites, individuals can either be susceptible (S), infected (I) or recovered (R). All partnerships engage in vaginal intercourse, and a fraction of partnerships will also have anal intercourse. We developed models including different transmission mechanisms, e.g. transmission by anal sex and autoinoculation between anatomic sites, and explored which mechanisms or combinations thereof fit the observed data best.
Results Most models did fit to the observed prevalence of male and female urogenital Ct: $13.6 \%$ (95\% CI: 10.7-17.2) and 13.0\% (95\% CI: 12.4-13.7), female anorectal Ct: 10.6\% (95\% CI: 8.0-13.9) and both sites: 9.9\% (95\% CI: 7.4-13.1). Models that assumed autoinoculation between anatomic sites fitted the data best, compared to models that focused on anal sex only. The model will be used to further determine the impact of testing strategies (i.e. universal irrespective of anal intercourse) and treatment strategies (i.e. azithromycin or doxycycline) on population prevalence.

Conclusions The results are suggestive of a Ct autoinoculation process between anatomic sites in women. This has potential consequences for future chlamydia control strategies including testing and treatment.

Disclosure of interest statement The National Institute of Public Health and the Environment is funded by the Ministry of Health, Welfare and Sport. The authors declare no conflict of interest.

\subsection{CLINICIAN-TAKEN EXTRA-GENITAL SAMPLES FOR GONORRHOEA AND CHLAMYDIA IN WOMEN COMPARED WITH SELF-TAKEN SAMPLES ANALYSED SEPARATELY AND SELF-TAKEN POOLED SAMPLES}

${ }^{1} \mathrm{JD}$ Wilson*, ${ }^{1} \mathrm{HE}$ Wallace, ${ }^{1} \mathrm{~J}$ Fisher, ${ }^{2} \mathrm{H}$ Ward, ${ }^{3} \mathrm{C}$ Hulme, ${ }^{4} \mathrm{MH}$ Wilcox. ${ }^{1}$ Leeds Centre for Sexual Health, Leeds Teaching Hospitals NHS Trust, UK; ${ }^{3}$ Academic Unit of Health Economics, University of Leeds, UK; ${ }^{4}$ Department of Clinical Microbiology, Leeds Teaching Hospitals NHS Trust, UK; ${ }^{2}$ Department of Infectious Disease Epidemiology, Imperial College, London, UK

\subsection{6/sextrans-2015-052270.95}

Background Extra-genital sampling (rectum and pharynx) using nucleic acid amplification tests is becoming increasingly important in women as vulvovaginal swabs (VVS) alone may miss infections. We compared clinician-taken extra-genital samples in women with self-taken samples analysed both separately and as pooled samples for accuracy and cost-effectiveness.

Methods Women attending a sexual health clinic were invited into a 'swab yourself' trial. Clinician and two self-samples (analysed separately and pooled) were taken from vulvovaginal, pharyngeal and rectal sites for gonorrhoea (NG) and chlamydia (CT) using AC2. Sampling order was randomised. Patient infected status was defined as at least two positive confirmed samples.

Results 402 women recruited January-March 2015. Overall prevalence: gonorrhoea $3.2 \%$ (rectal 2.7\%, pharyngeal $1.5 \%$ ), chlamydia $13.7 \%$ (rectal $12.9 \%$, pharyngeal $3.2 \%$ ). One NG case $(7.7 \%)$ and 7 CT cases (12.7\%) were VVS negative.

\begin{tabular}{lllll}
\hline & Sensitivity & Specificity & PPV & NPV \\
\hline NG Rectal Clinician & $100 \%$ & $100 \%$ & $100 \%$ & $100 \%$ \\
NG Rectal Self & $100 \%$ & $100 \%$ & $100 \%$ & $100 \%$ \\
NG Pharynx Clinician & $83.3 \%$ & $100 \%$ & $100 \%$ & $99.8 \%$ \\
NG Pharynx Self & $100 \%$ & $100 \%$ & $100 \%$ & $100 \%$ \\
NG Self Pooled & $100 \%$ & $100 \%$ & $100 \%$ & $100 \%$ \\
CT Rectal Clinician & $98.1 \%$ & $100 \%$ & $100 \%$ & $99.7 \%$ \\
CT Rectal Self & $100 \%$ & $99.7 \%$ & $98.1 \%$ & $100 \%$ \\
CT Pharynx Clinician & $92.3 \%$ & $99.7 \%$ & $92.3 \%$ & $99.7 \%$ \\
CT Pharynx Self & $84.6 \%$ & $100 \%$ & $100 \%$ & $99.5 \%$ \\
CT Self Pooled & $98.2 \%$ & $99.4 \%$ & $96.4 \%$ & $99.7 \%$ \\
\hline
\end{tabular}

